\title{
Reflets
}

Revue d'intervention sociale et communautaire

\section{Les principes d'intervention à la Table féministe francophone de concertation provinciale de l'Ontario}

\section{Lyne Bouchard}

Volume 9, numéro 1, printemps 2003

Le genre en contexte : pratiques sociales et représentations

URI : https://id.erudit.org/iderudit/010869ar

DOI : https://doi.org/10.7202/010869ar

Aller au sommaire du numéro

Éditeur(s)

Reflets : Revue ontaroise d'intervention sociale et communautaire

ISSN

1203-4576 (imprimé)

1712-8498 (numérique)

Découvrir la revue

Citer cet article

Bouchard, L. (2003). Les principes d'intervention à la Table féministe francophone de concertation provinciale de l'Ontario. Reflets, 9(1), 216-221.

https://doi.org/10.7202/010869ar

Tous droits réservés (C) Reflets : Revue ontaroise d'intervention sociale et communautaire, 2002
Ce document est protégé par la loi sur le droit d'auteur. L'utilisation des services d'Érudit (y compris la reproduction) est assujettie à sa politique d'utilisation que vous pouvez consulter en ligne.

https://apropos.erudit.org/fr/usagers/politique-dutilisation/ 


\title{
Les principes d'intervention à la Table féministe francophone de concertation provinciale de l'Ontario
}

\author{
Lyne Bouchard \\ Coopérative Convergence
}

\section{Introduction}

Cet article a été rédigé suite à la présentation d'une communication effectuée dans le cadre de l'ouverture du colloque du Réseau des chercheures féministes de l'Ontario français tenu au Collège universitaire de Glendon à Toronto du 19 au 21 avril 2002.

Il tente d'expliquer le type d'intervention privilégiée par la Table féministe francophone de concertation provinciale de l'Ontario (TFFCPO). Deux exemples de projets de recherche d'envergure de la TFFCPO soit action sur le parrainage ${ }^{1}$ publié $^{2}$ en 2001 et projet santé2 , 1996-1997, illustrent l'intervention de cette dernière. De plus, d'autres projets sont exposés et permettent d'appuyer le fondement des principes d'intervention de la TFFCPO. Ces projets, sans être de recherche, s'inspirent des principes de recherche action. Ils consistent entre autres, en la publication d'une série de onze feuillets ${ }^{3}$ dans le cadre de la Marche mondiale des femmes, en un projet intitulé «Les droits à 
l'égalité des Franco-Ontariennes $»^{4}$ et en un autre de sensibilisation au sujet du travail obligatoire.

\section{Contexte de l'intervention à la TFFCPO}

La Table féministe francophone de concertation provinciale de l'Ontario a été créée lors du premier colloque sur l'intervention féministe organisé par le Réseau des chercheures féministes de l'Ontario français qui s'est déroulé en février 1992 à l'Université Laurentienne, à Sudbury.

La TFFCPO se voulait un lieu de prise de conscience de la diversité des préoccupations des femmes, tant dans le fonctionnement du groupe que dans les projets mis de l'avant. Cet espace pour les femmes a été mis sur pied pour fournir un outil de revendication politique et améliorer les conditions de vie des femmes. La structure et la culture organisationnelle se veut transversale et souple ${ }^{5}$. Elle cherche à développer un concept de porte-parole multiples et fonctionne à l'aide de comités autonomes.

Au fil des années, la TFFCPO a développé une démarche d'intervention à trois volets, l'information/formation, la consultation et la concertation/action

\section{Information/formation}

Souvent à la source d'un projet ou d'une intervention, le volet information/formation vise trois grands objectifs, dont l'appropriation de l'information. Dans Conditions de possibilité des services de santé et de services sociaux en français en Ontario : un enjeu pour les femmes par exemple, les groupes membres de la TFFCPO souhaitaient intervenir dans le dossier sur lequel ils possédaient très peu de connaissances. L'information sur les difficultés vécues 
par les femmes pour recevoir un service n'étant pas colligée, il était difficile de planifier une intervention sérieuse sur la question. C'est la raison pour laquelle le comité santé de la TFFCPO a décidé en 1996 de mettre en place une équipe de huit chercheures ${ }^{6}$ afin d'effectuer une recherche-action auprès des services de santé, des services sociaux et également auprès des femmes. À l'étude, les dynamiques politiques de prestations de services de même que la dynamique de l'utilisation des services pour les usagères.

Dans «Les droits à l'égalité des Franco-Ontariennes », comme autre exemple, le volet information/formation a joué un rôle d'informateur via la formation d'éducation juridique populaire offerte au début du projet. Cette formation amenait les participantes à comprendre leurs droits relatifs à l'article 15 de la Charte canadienne des droits et libertés, en leur fournissant une base solide de connaissances leur permettant d'évaluer l'impact des programmes gouvernementaux sur leurs droits.

Un autre projet axé sur l'information, la série de onze feuillets produits dans le cadre de la Marche mondiale des femmes en 2000 , vise à sensibiliser ces dernières sur divers sujets. On peut conclure que le volet d'intervention (information/formation) cherche à informer tant les leaders du mouvement féministe que la population féminine en général sur un sujet leur permettant une meilleure intervention. Les moyens utilisés sont la recherche, la formation ou les campagnes de sensibilisation.

\section{Consultation}

Le volet consultation se trouve souvent à la deuxième étape d'un projet ou d'une intervention et vise principalement à connaitre les besoins et les intérêts des femmes. Si on prend pour exemple le projet de recherche sur la santé, une équipe de chercheures a interrogé plus de 36 femmes. D'une durée d'environ deux heures, les entrevues permettaient de mieux comprendre le vécu des femmes quant à leur santé et leur expérience auprès des services 
de santé. Le volet consultation du projet "Les droits à l'égalité des Franco-Ontariennes » démontrait clairement que l'égalité est loin d'être chose faite pour les femmes. Les sessions de consultation visaient à identifier les pratiques des gouvernements provincial et fédéral portant atteinte ou faisant obstacle aux droits à l'égalité garantis dans la Charte.

Ce volet d'intervention nourrit la TFFCPO lorsqu'elle prépare l'" argumentaire " de son action en lui permettant, grâce aux diverses consultations, de toujours s'inspirer de la réalité que vivent les femmes dans leur quotidien.

\section{Concertation/action}

Le volet concertation/action, souvent en dernière phase d'un projet ou d'une intervention, permet de déterminer les stratégies d'action et d'agir de façon efficace dans un dossier.

Prenant pour exemple Qui prend mari, prend pays, une rencontre de concertation a eu lieu à la fin de ce projet de recherche. Les participantes étaient, pour la plupart, des femmes immigrantes parrainées et des intervenantes travaillant auprès de la clientèle immigrante en Ontario. À cette rencontre, les résultats de la recherche ont été présentés et une stratégie d'action a été élaborée. Les participantes ont décidé de créer un organisme provincial, soit le Mouvement ontarien pour les femmes immigrantes francophones (MOFIF) chargé d'assumer les suites de la rencontre et d'assurer un plan d'action.

Pour ce qui est du projet de recherche sur la santé, le comité santé de la TFFCPO a organisé un forum provincial de concertation pour assurer un suivi à la recherche-action. FEMMES pour la santé a été mis en place lors de cette rencontre. Le groupe s'est impliqué dans divers dossiers et au sein de tables de concertation sur la santé. Il est présentement à la recherche de fonds pour concrétiser les recommandations de la recherche. 


\section{Conclusion}

La création du Mouvement ontarien des femmes immigrantes francophones (MOFIF) est un des aboutissants du projet "Les droits à l'égalité des Franco-Ontariennes " réalisé en 1997. Suite à une tournée provinciale orchestrée par la TFFCPO, le volet concertation du projet a donné priorité à la recherche sur les femmes immigrantes et le parrainage, sur la violence faite aux femmes $^{7}$ et sur le travail obligatoire. Un projet de recherche sur le parrainage a été effectué et lors du volet concertation de la recherche-action, les intervenantes auprès des femmes immigrantes ont créé le MOFIF rassemblant les groupes de femmes des communautés ethnoculturelles et raciales ainsi que les intervenantes travaillant auprès des femmes immigrantes.

Par le volet concertation du projet de recherche-action en matière de santé en 1998, la TFFCPO a créé FEMMES pour la santé. Ce comité est responsable de faire avancer les recommandations et de donner suite à la recherche.

Quoique les volets d'intervention soient interdépendants, ils peuvent être séquentiels ou s'entrecouper. Les principes d'intervention de la TFFCPO lui permettent non seulement d'ancrer son action dans la réalité des femmes mais aussi d'aller au-delà de ses limites en développant ses connaissances dans des domaines précis et parfois même ses compétences. On peut constater par les exemples cités que l'intervention exercée par la TFFCPO vise à mettre en place une organisation apprenante s'inscrivant dans des pratiques concrètes d'apprentissage, de transfert d'apprentissages tout autant que de savoirs.

\section{Notes}

1. Côté,Andrée, Kérisit, Michelle et Côté, Marie-Louise, Qui prend pays... l'impact du parrainage sur les droits à l'égalité des femmes immigrantes, Condition féminine Canada, Ottawa, 2001

2. Andrew, C., Bouchard, L., Boudreau, F., Cardinal, L., Farmer, D., Kérisit, M., Lemire, D., en collaboration avec Adam, D., Culligan, D., Dallaire, H. et Rochon-Ford,A., Conditions de possibilité 
des services de santé et de services sociaux en français en Ontario : un enjeu pour les femmes, Table féministe francophone de concertation provinciale de l'Ontario, Ottawa, 1997.

3. L'État engendre la pauvreté; La pauvreté sans frontières; La sécurité sociale : un filet troué; La recherche d'emploi : les obstacles; La maternité; Lesbiennes et citoyennes;Violence conjugale; Violence sexuelle;Violence contre les femmes par l'État; Garde légale : réforme de la Loi sur le divorce.

4. Côté, Andrée, Les droits à l'égalité garantis à l'article 15 de la Charte canadienne des droits et libertés, Table féministe francophone de concertation provinciale de l'Ontario, Ottawa, septembre 1996.

5. Bouchard, Lyne, Affirmation politique des femmes au sein du milieu associatif, Reflets, vol. 3, no. 2, automne 1997.

6. L'équipe était composée de trois chercheures provenant de l'Université d'Ottawa, deux provenaient du Collège universitaire de Glendon, une de l'Office des Affaires francophones de l'Ontario et de deux chercheures indépendantes.

7. Ce dossier est confié à l'Action ontarienne contre la violence faite aux femmes regroupant les organismes et les intervenantes francophones qui luttent contre la violence. 